\title{
MESSAGE
}

\section{From the Executive}

I have spoken before about where we are in the HIV epidemic in South Africa (SA) today. The heady days when there were scientific breakthroughs every few months are over. The excitement surrounding increased treatment access, improved antiretroviral therapy (ART) regimens and nurseinitiated management of antiretroviral therapy (NIMART) have settled; HIV no longer makes the news every other day. What we have achieved is truly remarkable: SA has the largest antiretroviral (ARV) programme in the world, with estimates of as many as 2.4 million people receiving ART. HIV healthcare workers can be proud of the contributions they have made - and continue to make - to lessen the burden of this disease.

Now that the excitement has passed, it is time to get on with the slog of rolling out and sustaining a massive treatment programme. What we are finding is that this may actually be our greatest challenge yet. In this issue of SAJHIVMED you will find an insert highlighting key points from a national survey conducted by the Stop Stock Outs Project assessing ARV and tuberculosis drug stockouts at the facility level. As you will see, the report found that the problem is far beyond previous estimates, and affects most provinces. The telephone survey, which took place during September and October 2013, obtained information from over 2000 health facilities. Around one in every five facilities in SA reported a stockout or shortage during the 90-day period covered in the survey. Free State, Limpopo and Mpumalanga provinces were the worst affected, with $54 \%, 41 \%$ and $26 \%$ of facilities affected, respectively. In $20 \%$ of facilities facing stockouts, patients were sent away with no medicine.

To some of you these results won't be surprising at all, as you are negotiating medicine shortages and stockouts on a daily basis. What the report makes clear is that stockouts are so common, that they represent a credible threat to the success of the national ARV programme.

The report is also a reminder that although the fight may be over, the battle is far from won. As we begin 2014 and prepare to mark ten years of ART in the public sector, we must celebrate what we have achieved, but not become complacent about where we need to be. As healthcare workers we must redouble our efforts and contribute our part to strengthening the health system, whether it's reporting medicine stockouts, corruption or inefficiencies within the system. What we have accomplished in ten years is no less than remarkable; what is possible for us to achieve in the next ten is truly inspiring.

\section{Francesca Conradie}

President

Southern African HIV

Clinicians Society

fconradie@witshealth.co.za

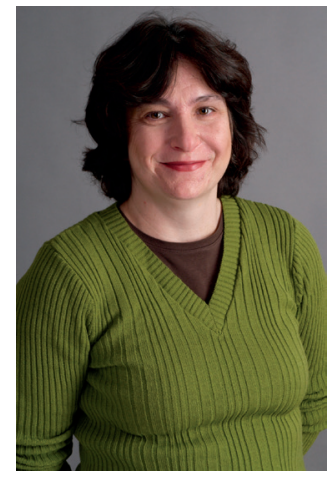

\section{Call for submissions}

\section{A decade of antiretroviral therapy in the public sector}

As 2014 marks the 10-year anniversary of the public sector rollout of antiretroviral therapy (ART) services in South Africa, SAJHIVMED is planning a special edition to reflect on the lessons learned and celebrate the achievements during this time.

As part of this, SAJHIVMED is calling for submissions from healthcare workers, policy makers and researchers towards this special edition. Contributions of all shapes and sizes are welcome. We are particularly interested in 'reflections' from clinicians on the ground - providing insight into the realities of delivering ART to increasing numbers of patients, often under difficult circumstances.

These submissions can take the form of editorials of 500 - 1000 words or longer, commenting on individual experiences of providing HIV care and treatment services at all levels of care. If you are interested, please submit these pieces by 15 January 2014 via the journal website (http://www.sajhivmed.org.za) or email the Editor directly (landon.myer@uct.ac.za). 